\title{
Electrochemical Oxidation of 2'-deoxyguanosine-5'-triphosphate on Ionic Liquid Modified Carbon Paste Microelectrode and its Sensitive Detection
}

\author{
Hongwei Gao, ${ }^{a}$ Yuanyuan Duan,,${ }^{\mathrm{b}} \mathrm{Li}$ Xu,,${ }^{\mathrm{b}}$ and Wei Sun ${ }^{\mathrm{b}, *}$ \\ ${ }^{a}$ Shandong Entry-Exit Inspection and Quarantine Bureau of People's Republic of China, \\ Qingdao 266002, P. R. China \\ ${ }^{\mathrm{b}}$ College of Chemistry and Molecular Engineering, Qingdao University of Science and Technology, \\ Qingdao 266042, P. R. China
}

RECEIVED JULY 20, 2010; REVISED DECEMBER 15, 2010; ACCEPTED DECEMBER 17, 2010

\begin{abstract}
Electrochemical oxidation of 2'-deoxyguanosine-5'-triphosphate (dGTP) was investigated on the ionic liquid modified carbon paste microelectrode by cyclic voltammetry in this paper. The carbon ionic liquid microelectrode (mCILE) was prepared with ionic liquid 1-ethyl-3-methylimidazolium ethylsulfate $\left([\mathrm{Emim}]\left[\mathrm{EtOSO}_{3}\right]\right)$ as the modifier. Then the electrochemical process of dGTP on the mCILE was recorded with the electrochemical parameters calculated and the oxidation mechanism discussed. Under the optimal conditions differential pulse voltammetric peak current was proportional to the dGTP concentration in the range from $1.0 \times 10^{-6} \mathrm{M}$ to $7.0 \times 10^{-4} \mathrm{M}$ with the linear regression equation as $\log \left(I_{\mathrm{pa}} / \mathrm{A}\right)=$ $0.77 \log \left(c / \mathrm{mol} \mathrm{dm}^{-3}\right)-4.082(\gamma=0.999)$. The proposed method showed good selectivity and stability without the interferences of coexisting substances. (doi: 10.5562/cca1737)
\end{abstract}

Keywords: 2'-deoxyguanosine-5'-triphosphate, carbon ionic liquid microelectrode, cyclic voltammetry, electrochemistry, electrooxidation

\section{INTRODUCTION}

2'-Deoxyguanosine-5'-triphosphate (dGTP) is the most important substance required for DNA synthesis and several normal cellular functions. For example the concentration of dGTP had an great impact on human and Chinese Hamster Ovary (CHO) telomerase activities, which could produce longer primer extension products with the increase of dGTP concentration over a range of $1.5-50 \mu \mathrm{M}^{1}{ }^{1}$ A kinetics shift of primers biphasic dissociation from slow phase to fast phase was observed in the presence of dGTP, which may implicate dGTP as a positive effector of translocation. ${ }^{2}$ Also the attack of cellular dGTP by $\mathrm{OH} \cdot$ radical can result in the oxidized 2'-deoxyguanosine-5'-triphosphate (oxo-dGTP), which is promutagenic due to its tendency to preferentially pair with adenosine over cytosine during DNA replication, leading to $\mathrm{G}: \mathrm{C} \rightarrow \mathrm{T}: \mathrm{A}$ transversions. ${ }^{3,4} \mathrm{Be}-$ cause the concentration of dGTP in the cytoplasm is very lower, ${ }^{5}$ sensitive and accurate methods are required for dGTP determination in biological samples. Electrochemical methods including voltammetry and amperometry have been exhibited the advantages such as wide dynamic range, low detection and ease of mi- niaturization. The electrochemistry of bases has been investigated on different kind of working electrodes. Xie et al. investigated the electrochemical behavior of guanine, guanosine, and guanosine monophosphate at redox polymer film modified indium tin oxide (ITO) electrode. ${ }^{6}$ Goyal et al. investigated the electrochemical oxidation mechanism of guanosine-5'-triphosphate (GTP $)^{7}$ and guanosine-5' ${ }^{\prime}$-monophosphate ${ }^{8}$ at a pyrolytic graphite electrode. They also used a nanogold modified ITO electrode for the simultaneous determination of guanosine and GTP in the human blood plasma samples $^{9}$. Zhang et al. described a novel PCR-electrochemical detection strategy using dGTP as a probe, which had a well-defined voltammetric oxidation peak at the short single-walled carbon nanotubes modified glassy carbon electrode. ${ }^{10}$

Ionic liquids (ILs) are a new kind of modifier in the carbon paste electrode (CPE), which have been exhibited many advantages such as high ionic conductivity, wide electrochemical windows and stable electrochemical responses. Preparation of carbon paste electrodes with ILs as a binder, which is often named as carbon ionic liquid electrode (CILE), has been reported by several groups. ${ }^{11-14}$ ILs-based carbon paste

* Author to whom correspondence should be addressed. (E-mail: sunwei@qust.edu.cn) 
electrodes show very sensitive and stable responses compared to pastes made of non-conductive binder such as liquid paraffin. The enhanced anti-fouling effect of the ILs paste electrode is another feature to be added to this attractive class of material. However, one major concern is the high background current which limited the analytical utility of these IL-based paste electrodes. One approach to minimize the background current and to increase the analytical signal-to-background ratio is made via decreasing the electrode active surface area and increasing mass transport rates by utilizing microelectrodes. El-Nour prepared a highly active carbon fiber ultramicroelectrode (CFE) as sensor in the sensitive determination of adenosine under physiological conditions. ${ }^{15}$ Oliveira-Brett used a carbon fiber microelectrode for the investigation on the electrooxidation mechanism of guanine and adenine. ${ }^{16}$ The reduced capacitive contribution of microelectrodes makes it possible to study the electron transfer (ET) reactions of these processes by means of fast scan rate cyclic voltammetry and to establish a more sensitive detection method by differential pulse voltammetry. Musameh et al. also fabricated different ILs modified carbon paste microelectrode and investigated the detection of different electroactive species, which exhibited the advantages of improved sensitivity, stability and reproducibility. ${ }^{17}$

In this paper a carbon ionic liquid microelectrode (mCILE) was first prepared with ionic liquid 1-ethyl-3methylimidazolium ethylsulfate ([Emim][EtOSO 3$])$. Electrochemical characteristics of mCILE were investigated by cyclic voltammetry and electrochemical impedance spectroscopy. Then the electrochemical behavior of dGTP on the mCILE was investigated carefully and further a sensitive electrochemical method for dGTP detection was established.

\section{EXPERIMENTAL}

\section{Reagents}

1-Ethyl-3-methylimidazolium ethylsulfate ([Emim] [EtOSO 3 , Hangzhou Kemer Chemical Limited Company, China) and graphite powder (average particle size $30 \mu \mathrm{m}$, Shanghai Colloid Chemical Plant, China) were used as received. 2'-deoxyguanosine-5'- triphosphate ( $\geqq 98 \%$, Shanghai Promega Biological Products Ltd., China) was supplied as buffer-free, ready-to-use $10.0 \mathrm{M}$ solution in water. A series of $0.2 \mathrm{M}$ Britton-Robinson (B-R) buffer solutions were adjusted with $0.5 \mathrm{M}$ $\mathrm{NaOH}$ and used as the supporting electrolyte. All the other chemicals were commercially available with analytical grade and used without further purification. All the solutions were prepared with doubly distilled water.

\section{Apparatus}

Electrochemical measurements including cyclic voltammetry (CV) and differential pulse voltammetry (DPV) were performed on a CHI 1210A electrochemical workstation (Shanghai Chenhua Co., China). Electrochemical impedance spectroscopy (EIS) was carried out with a CHI 750B electrochemical workstation (Shanghai Chenhua Co., China). A conventional threeelectrode system was employed with a homemade mCILE (300 $\mu \mathrm{m}$ diameter) working electrode, an $\mathrm{Ag} \mid \mathrm{AgCl}$ reference electrode and a platinum wire auxiliary electrode. All the following potentials reported in this work were versus the $\mathrm{Ag} \mid \mathrm{AgCl}$ reference electrode.

\section{Procedure}

$40 \mu \mathrm{L}$ of [Emim][EtOSO 3 ], $100 \mathrm{mg}$ of paraffin oil, and $500 \mathrm{mg}$ of graphite powder were mixed and ground carefully to form a homogeneous paste, which was then packed at the end of a pipette tip (diameter $300 \mu \mathrm{m}$ ) to a depth of $3 \mathrm{~mm}$. Electrical contact was achieved via a copper wire to the end of the tip. The resulting microelectrode was denoted as mCILE. For comparison the carbon paste microelectrode (mCPE) was prepared by the similar procedure by mixing $500 \mathrm{mg}$ of graphite powder with $100 \mathrm{mg}$ of paraffin oil. Prior to use, the surface of the well-prepared microelectrode was smoothed on a weighing paper.

\section{RESULTS AND DISCUSSION}

\section{Characterization of the Microelectrodes}

Electrochemical impedance spectroscopy (EIS) can give information on the interface changes of the modified electrode. The semicircle diameter of well-conducting substrates equals to the electron transfer resistance $\left(R_{\mathrm{et}}\right)$. The value of $R_{\text {et }}$ varies when different substances are adsorbed or modified on the electrode surface. By using $1.0 \times 10^{-3} \mathrm{M}$ solution of $\left[\mathrm{Fe}(\mathrm{CN})_{6}\right]^{3-/ 4-}$ as redox probe, the EIS of different electrodes were recorded with the results shown in Figure 1a. On the traditional mCPE a big semicircle domain appeared, indicating the existence of a big electron transfer resistance (curve 1). The result was due to the presence of non-conductive paraffin oil in the carbon paste. While on the MCILE a nearly straight line appeared and the $R_{\text {et }}$ value was much smaller than that of $\mathrm{mCPE}$ (curve 2), indicating that the presence of high ionic conductive $\left[\right.$ Emim] $\left[\mathrm{EtOSO}_{3}\right]$ in the carbon paste facilitated the electron transfer of the electrochemical probe. Figure $1 \mathrm{~b}$ showed cyclic voltammetric responses of $\mathrm{mCPE}$ and mCILE in $1.0 \times 10^{-3} \mathrm{M}$ $\mathrm{K}_{3}\left[\mathrm{Fe}(\mathrm{CN})_{6}\right]$ and $0.5 \mathrm{M} \mathrm{KCl}$ mixture solution at the scan rate, $v$ of $500 \mathrm{mV} \mathrm{s}^{-1}$. On the $\mathrm{mCPE}$ (curve 1), the 

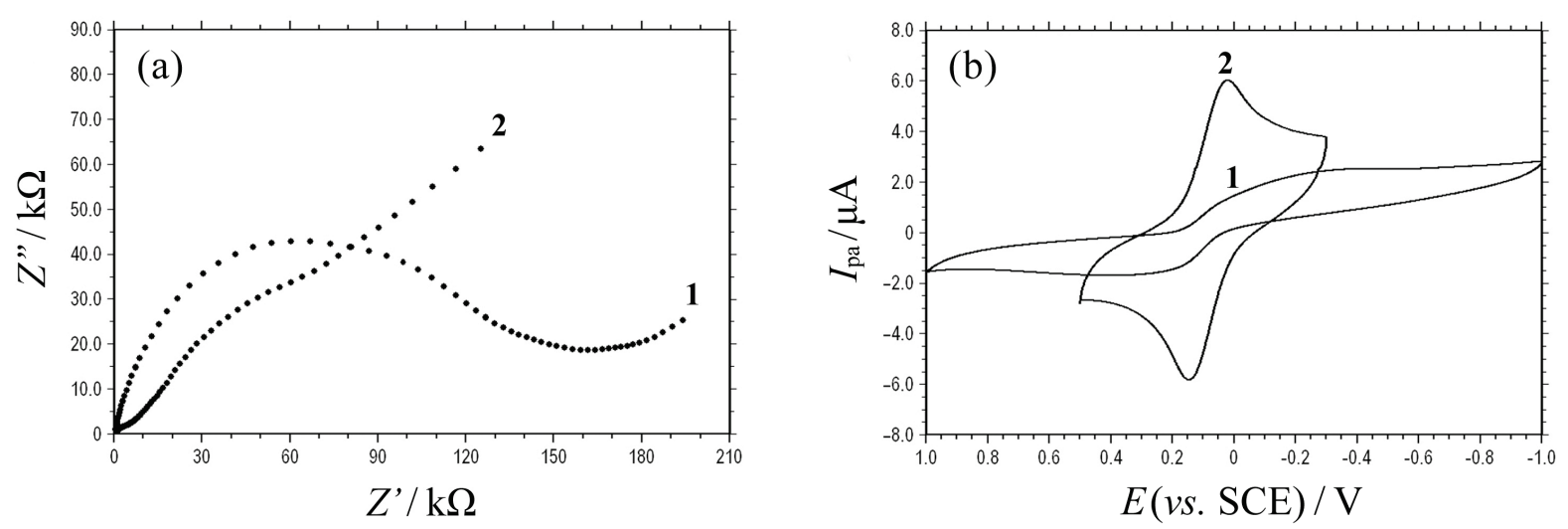

Figure 1. Electrochemical impedance spectra (a) for mCPE (1) and mCILE (2) in a mixture $1.0 \times 10^{-3} \mathrm{M}$ solution of $\mathrm{K}_{3}\left[\mathrm{Fe}(\mathrm{CN})_{6}\right]^{3-/ 4}$ and $0.5 \mathrm{M} \mathrm{KCl}$ with the frequencies swept from $10^{4}$ to $0.1 \mathrm{~Hz}$. Cyclic voltamograms (b) of mCPE (1) and mCILE (2) in mixture solution of $1.0 \times 10^{-3} \mathrm{M} \mathrm{K}_{3}\left[\mathrm{Fe}(\mathrm{CN})_{6}\right]+0.5 \mathrm{M} \mathrm{KCl}$ at the scan rate of $500 \mathrm{mV} \cdot \mathrm{s}^{-1}$.

peak-to-peak potential separation $\left(\Delta E_{\mathrm{p}}\right)$ was calculated as $450 \mathrm{mV}$ with deformed curve, corresponding to a quasi-reversible electron transfer process. While on the mCILE (curve 2), the $\Delta E_{\mathrm{p}}$ was decreased to $124 \mathrm{mV}$, indicating a more reversible electron transfer process. Furthermore, the redox peak currents on the mCILE were 2.23-fold higher than that of the $\mathrm{mCPE}$, indicating the high conductivity of the electrode interface.

All the above experimental results exhibited the superiority of mCILE to mCPE in terms of improved reversibility and enhanced sensitivity, which were attributed to the use of high conductive ILs as a modifier. On the mCILE the redox peak current increased gradually with the scan rate, $v$ in the range from 10 to $500 \mathrm{mV} \mathrm{s}^{-1}$. A linear relationship was got between the redox peak current with the square root of scan rate, indicating that the electrochemical process was diffusion-controlled. Two linear regression equations were calculated as $I_{\mathrm{pc}} / \mathrm{mA}=6.229 v^{1 / 2}+0.2147(\gamma=0.998)$ for mCILE and

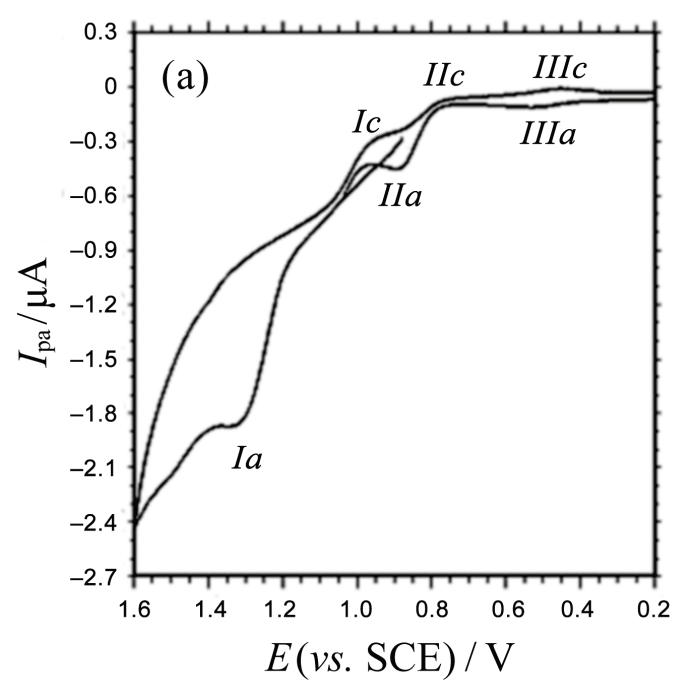

$I_{\mathrm{pc}} / \mathrm{mA}=2.042 v^{1 / 2}+0.6735 \quad(\gamma=0.987)$ for $\mathrm{mCPE}$. According to the Randles-Sevcik equation, ${ }^{18} I_{\mathrm{p}}=$ $2.69 \times 10^{5} n^{3 / 2} A D^{1 / 2} c v^{1 / 2}$, where $n$ is the number of electrons transferred, $A$ is the electrode area (expressed in $\mathrm{cm}^{2}$ ), $c$ is the concentration (expressed in mol $\mathrm{dm}^{-3}$ ), $D$ is the diffusion coefficient of $\left[\mathrm{Fe}(\mathrm{CN})_{6}\right]^{3-}\left(6.5 \times 10^{-6}\right.$ $\mathrm{cm}^{2} \mathrm{~s}^{-1}$ ) and $v$ is the scan rate (expressed in $\mathrm{V} \mathrm{s}^{-1}$ ). The electrode effective area of the mCILE is estimated to be $0.908 \mathrm{~mm}^{2}$, which was about 3 times higher than that of the $\mathrm{mCPE}\left(0.298 \mathrm{~mm}^{2}\right)$. So the presence of IL in the carbon paste electrode greatly improved the electrochemical performances.

\section{Cyclic Voltamograms of 2'-deoxyguanosin-5'-tri- phosphate}

Typical cyclic voltamograms of $5.0 \times 10^{-4} \mathrm{M}$ dGTP on mCILE and mCPE were recorded with the results shown in Figure 2. As shown in Figure 2a, on mCPE a

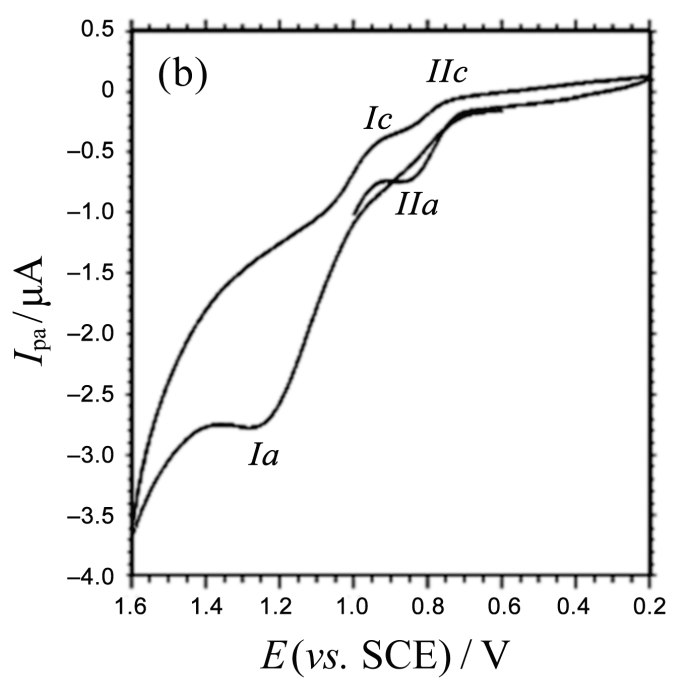

Figure 2. Cyclic voltamograms of $5.0 \times 10^{-4} \mathrm{M}$ dGTP on mCPE (a) and mCILE (b) in B-R buffer solution of $\mathrm{pH}=4.0$ at the scan rate, $v$ of $200 \mathrm{mV} \mathrm{s}^{-1}$. 
single well-defined oxidation peak (Ia) appeared at $E=+1.323 \mathrm{~V}$ with the oxidation peak current as $38.38 \mathrm{nA}$ when the sweep was initiated in the positive direction. In the reverse sweep three cathodic peaks Ic $(0.969 \mathrm{~V})$, IIc $(0.782 \mathrm{~V})$ and IIIc $(0.535 \mathrm{~V})$ appeared. In the continuous (second) sweep to the positive direction another two oxidation peaks IIa $(0.879 \mathrm{~V})$ and IIIa $(0.447 \mathrm{~V})$ appeared. The phenomena was very similar to the electrooxidation of GTP, ${ }^{7}$ guanoside-5'-monophosphate $(\mathrm{GMP})^{8}$ or 2'-deoxyguanosine-5'-monophosphate $(\mathrm{dGMP})^{19}$ on the pyrolytic graphite electrode. Goyal et al. had widely investigated the oxidation mechanisms of purine based and their nucleosides or nucleotides on the solid electrode with different methods such as cyclic voltammetry, spectral studies, bulk electrolysis and related techniques. The detailed redox mechanism for the purine nucleotide was also proposed with the final products identified. While on mCILE a big and welldefined oxidation peak (Ia) appeared with the oxidation peak potential as $1.254 \mathrm{~V}$ and the oxidation peak current as $73.46 \mathrm{nA}$ on the first positive sweep (Figure 2b). Based on the electro-oxidation mechanism of guanine or guanosine with the similar molecular structure, ${ }^{16,20}$ this oxidation peak can be attributed to the oxidation of dGTP to a free radical moiety and further form a diimine specie. In the reverse sweep two cathodic peaks of Ic $(0.943 \mathrm{~V})$ and IIc $(0.756 \mathrm{~V})$ appeared. In the successive second sweep towards positive potential another well-defined oxidation peak IIa $(0.835 \mathrm{~V})$ appeared. The result also proved the complicated oxidation mechanism involved in the reduction and subsequent oxidation of the species generated during the voltammetric sweep. Also it can be seen that the oxidation peak currents increased with the decrease of the oxidation peak potential, indicating the electrocatalytic activity of mCILE.

The influence of buffer $\mathrm{pH}$ on the voltammetric responses of different redox peaks on MCILE was further investigated in the $\mathrm{pH}$ range from 2.0 to 7.0. In all cases the redox peak potential was $\mathrm{pH}$ dependent and shifted with the change of buffer $\mathrm{pH}$. The linear relationships of $E_{\mathrm{p}} v s . \mathrm{pH}$ were summarized in Table 1 with different $\mathrm{pH}$ range. From the slope it can be concluded that protons took part in the electrode reaction. The

Table 1. Influence of buffer $\mathrm{pH}$ on the redox peak potential of dGTP

\begin{tabular}{ccc}
\hline Peak & $\begin{array}{c}\mathrm{pH} \\
\text { range }\end{array}$ & $\begin{array}{c}\text { Linear regression equations } \\
\left(E_{\mathrm{p}} \text { is expressed in V }\right)\end{array}$ \\
\hline Ia & $2.0-7.0$ & $E_{\mathrm{p}}=-0.041 \mathrm{pH}+1.43(\gamma=0.967)$ \\
Ic & $3.0-7.0$ & $E_{\mathrm{p}}=-0.056 \mathrm{pH}+1.11(\gamma=0.995)$ \\
IIc & $2.0-4.0$ & $E_{\mathrm{p}}=-0.040 \mathrm{pH}+0.85(\gamma=0.979)$ \\
IIa & $2.0-7.0$ & $E_{\mathrm{p}}=-0.078 \mathrm{pH}+1.13(\gamma=0.975)$ \\
\hline
\end{tabular}

results were similar to the reports of electrooxidation dGMP. ${ }^{19}$

The effect of scan rate on the redox peak potential $\left(E_{\mathrm{p}}\right)$ and peak current $\left(I_{\mathrm{p}}\right)$ were further investigated in the scan rate range from 10 to $400 \mathrm{mV} \mathrm{s}^{-1}$. With the increase of scan rate the oxidation peak potentials shifted towards more positive directions and the reduction peak potentials moved towards more negative directions. The relationships of the redox peak responses with scan rate were constructed with the results summarized in Table 2. The peak current showed a good linear relationship with scan rate, indicating an EC nature of the reaction, in which the electrode reaction was coupled with an irreversible follow-up chemical reaction as well as an adsorption-controlled process. Based on the Laviron's equations: ${ }^{21}$

$$
\begin{gathered}
E_{\mathrm{pa}} \mathrm{v}=E^{\circ}+\frac{R T}{(1-\alpha) n F} \\
E_{\mathrm{pc}} v=E^{\circ}+\frac{R T}{\alpha n F}
\end{gathered}
$$

The electrochemical parameters such as electron transfer number $(n)$, electron transfer coefficient $(\alpha)$ and electrode reaction standard rate constant $\left(k_{\mathrm{s}}\right)$ of different steps were calculated, respectively, with the results listed in Table 2. Because the first oxidation peak (Ia) is big and stable, so it was selected for the further dGTP detection. Also due to the adsorption behavior of dGTP on the microelectrode, the electrode cannot be directly used after the each measurement. The microelectrode can be regenerated by simply smoothing on a weighing paper.

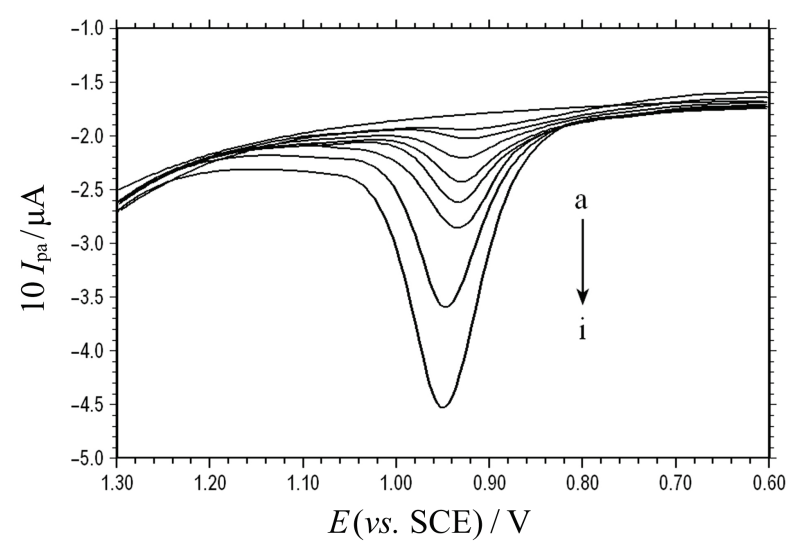

Figure 3. DPV curves of different concentrations dGTP on mCILE (from a to i, expressed in $\mathrm{mol} \mathrm{dm}^{-3}: 0,4.0 \times 10^{-6}$, $7.0 \times 10^{-6}, 1.0 \times 10^{-5}, 4.0 \times 10^{-5}, 7.0 \times 10^{-5}, 1.0 \times 10^{-4}, 4.0 \times 10^{-4}$, $7.0 \times 10^{-4}$ ) in B-R buffer of $\mathrm{pH}=4.0$. 
Table 2. Electrochemical parameters of dGTP on the mCILE

\begin{tabular}{|c|c|c|c|c|c|}
\hline Peak & \multicolumn{2}{|c|}{ Linear regression equations $^{(a)}$} & $n$ & $\alpha$ & $k_{\mathrm{s}}$ \\
\hline \multirow{2}{*}{ Ia } & $E_{\mathrm{p}}=0.0587 \ln v+1.372$ & $(\gamma=0.999)$ & \multirow{2}{*}{1.28} & \multirow{2}{*}{0.71} & \multirow{2}{*}{$3.91 \times 10^{-6}$} \\
\hline & $I_{\mathrm{p}}=2792 v+228.5$ & $(\gamma=0.991)$ & & & \\
\hline \multirow{2}{*}{ Ic } & $E_{\mathrm{p}}=0.0189 \ln v+0.991$ & $(\gamma=0.995)$ & \multirow{2}{*}{1.66} & \multirow{2}{*}{0.82} & \multirow{2}{*}{$3.30 \times 10^{-5}$} \\
\hline & $I_{\mathrm{p}}=621.8 v+59.26$ & $(\gamma=0.990)$ & & & \\
\hline \multirow{2}{*}{ IIc } & $E_{\mathrm{p}}=0.0159 \ln v+0.78$ & $(\gamma=0.996)$ & \multirow{2}{*}{2.08} & \multirow{2}{*}{0.78} & \multirow{2}{*}{$1.27 \times 10^{-5}$} \\
\hline & $I_{\mathrm{p}}=336.5 v+34.71$ & $(\gamma=0.993)$ & & & \\
\hline \multirow{2}{*}{ IIa } & $E_{\mathrm{p}}=0.0389 \ln v+1.178$ & $(\gamma=0.996)$ & \multirow{2}{*}{2.00} & \multirow{2}{*}{0.25} & \multirow{2}{*}{$2.50 \times 10^{-6}$} \\
\hline & $I_{\mathrm{p}}=780.7 v+72.03$ & $(\gamma=0.986)$ & & & \\
\hline
\end{tabular}

(a) $E_{\mathrm{p}}$ is expressed in $\mathrm{V}, I_{\mathrm{p}}$ in $\mathrm{nA}, v$ in $\mathrm{V} \mathrm{s}^{-1}$.

\section{DPV detection}

Under the optimal conditions dGTP was detected by more sensitive differential pulse voltammetry (DPV) with the typical voltamograms shown in Figure 3. A linear relationship could be established between the oxidation peak current (Ia) and the dGTP concentration in the range of $1.0 \times 10^{-6}-7.0 \times 10^{-4} \mathrm{M}$. The linear regression equation was calculated as $\log I_{\mathrm{pa}} / \mathrm{A}=0.77$ $\log \left(c / \mathrm{mol} \mathrm{dm}^{-3}\right)-4.082(\gamma=0.999)$ with the detection limit as $1.0 \times 10^{-6} \mathrm{~mol} \mathrm{dm}^{-3}(\mathrm{~S} / \mathrm{N}=3)$.

To estimate the reproducibility of the proposed method, the relative standard deviation (RSD) of eight times parallel measurements of $4.0 \times 10^{-4} \mathrm{M}$ dGTP at the mCILE was calculated to be $\pm 4.2 \%$, which demonstrated the good reproducibility of the method. The electrode-to-electrode repeatability was examined on three mCILEs constructed individually with the RSD value as $\pm 7.3 \%$, indicating the excellent repeatability of the microelectrode. Because the IL used is hydrophilic, so the stability of mCILE was investigated carefully. A stable electrochemical response was obtained after scanning in the ferricyanide solution for 80 circles at the scan rate of $500 \mathrm{mV} \mathrm{s}^{-1}$, indicating the stability of the mCILE in the solution. After the modified electrode was stored for 3 weeks, only a small decrease of peak current with a relative standard deviation (RSD) of $4.5 \%$ for $4.0 \times 10^{-4}$ mol dm${ }^{-3} \mathrm{dGTP}$ was observed, which exhibited the long storage stability of the microelectrode.

\section{CONCLUSIONS}

In this paper a carbon ionic liquid microelectrode (mCILE) was fabricated and used for the sensitive detection of dGTP by differential pulse voltammetry. Electrochemical behaviors of dGTP on mCILE was investigated by cyclic voltammetry with a series of redox peaks appeared, which was due to the initial oxidation of dGTP to a diimine species with following chemical reaction to give different products. Due to the presence of IL in the electrode, the electrochemical response was increased, which was ascribed to the electrocatalytic ability of mCILE. Compared with early reports about electrooxidation of $\mathrm{dGMP}^{19}$ or $\mathrm{GTP}^{7}$ on the pyrolytic graphite electrode, the initial oxidation peak (Ia) appeared on the cyclic voltamogram was contributed to the one electron and one proton oxidation step of guanine group to give a free radical moiety, which was occurred from the $\mathrm{CH}$ group at position 8 . The free radical formed could give a diimine species or take place different following chemical reactions with different kinds of oxidation products formed. Due to the presence of ribose or ribosephosphate units in the molecular structure of purine nucleotide, the oxidation mechanism was also affected with different products formed. Based on the oxidation reaction dGTP can be detected in the concentration range from $1.0 \times 10^{-6} \mathrm{M}$ to $7.0 \times 10^{-4} \mathrm{M}$ with the detection limit of $1.0 \times 10^{-6} \mathrm{~mol}$ $\mathrm{dm}^{-3}$. So the proposed microelectrode had the potential applications in the sensitive dGTP detection.

Acknowledgements. We are grateful to the financial support of the Science Foundation of General Administration of Quality Supervision, Inspection and Quarantine of the People's Republic of China (2010IK125) and the Shandong Province Natural Science Foundation (ZR2009BL017).

\section{REFERENCES}

1. P. Maine, S. F. Chen, and B. Windle, J. Biochem. 38 (1999) 15325-15332.

2. P. W. Hammond and T. R. Cech, J. Biochem. 37 (1998) 5162 5172 .

3. H. Hayakawa, A. Taketomi, K. Sakumi, M. Kuwano, and M. Sekiguchi, J. Biochem. 34 (1995) 89-95.

4. K. C. Cheng, D. S. Cahill, H. Kasai, S. Nishimura, and L. A. Loeb, J. Biol. Chem. 267 (1992) 166-172.

5. A. Kornberg and T. Baker, DNA Replication, W. H. Freeman and Company, New York, 1992.

6. H. Xie, D. W. Yang, A. Heller, and Z. Q. Gao, Biophys. J. 92 (2007) 70-72.

7. R. N. Goyal and A. Tyagi, Anal. Bioanal. Chem. 382 (2005) 1683-1690.

8. R. N. Goyal, B. K. Puri, and N. Jain, J. Chem. Soc. Perkin Trans. 2 (2001) 832-837. 
9. R. N. Goyal, M. Oyama, and A. Tyagi, Anal. Chim. Acta $\mathbf{5 8 1}$ (2007) 32-36.

10. X. Z. Zhang, S. F. Liu, K. Jiao, H. W. Gao, and Y. J. Shi, Analyst 133 (2008) 1729-1735.

11. H. T. Liu, P. He, Z. Y. Li, C. Y. Sun, L. H. Shi, Y. Liu, G. Y. Zhu, and J. H. Li, Electrochem. Commun. 7 (2005) 1357-1363.

12. N. Maleki, A. Safavi, and F. Tajabadi, Anal. Chem. 78 (2006) 3820-3826.

13. W. Sun, R. F. Gao, and K. Jiao, J. Phys. Chem. B 111 (2007) $4560-4567$.

14. G. Shul, J. Sirieix-Plenet, L. Gaillon, and M. Opallo, Electrochem. Commun. 8 (2006) 1111-1114.
15. K. A. El-Nour and A. Brajter-Toth, Electroanalysis 12 (2000) $805-810$.

16. M. Oliveira-Brett, V. Diculescu, and J. A. P. Piedade, Bioelectrochemistry 55 (2002) 61-62.

17. M. Musameh and J. Wang, Anal. Chim. Acta. 606 (2008) 45-49.

18. A. J. Bard and L. R. Faulkner, Electrochemical Methods, $2^{\text {nd }}$ ed., Wiley, New York, 2001, p 231.

19. R. N. Goyal, S. M. Sondhi, and A. M. Lahoti, New J. Chem. 29 (2005) 587-595

20. R. N. Goyal, N. Jain, and D. K. Garg, Bioelectrochem. Bioenerg. 43 (1997) 105-114.

21. E. Laviron, J. Electroanal. Chem. 100 (1979) 263-270. 